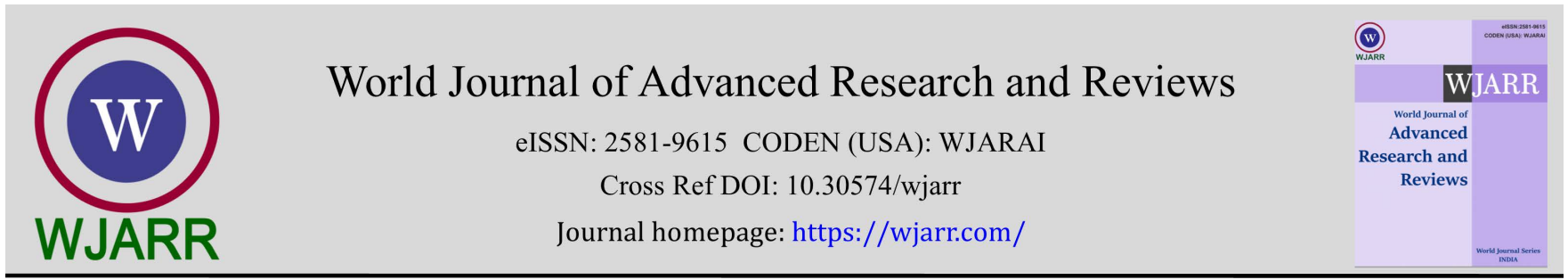

(CASE REPORT)

Check for updates

\title{
Fahr syndrome, rare cause of neuropsychiatric manifestations in children: A two cases report
}

\author{
Karima Larbi Ouassou *, Anas Ayad, Amale Hassani, Rachid Abilkassem and Aomar Agadr \\ Department of pediatrics- Mohammed V Military Instruction Hospital - Rabat, Morocco.
}

World Journal of Advanced Research and Reviews, 2021, 12(01), 268-274

Publication history: Received on 04 September 2021; revised on 08 October 2021; accepted on 10 October 2021

Article DOI: https://doi.org/10.30574/wjarr.2021.12.1.0510

\begin{abstract}
Fahr syndrome is a rare anatomo-clinical entity in pediatrics. Associating calcifications of the basal ganglia with phospho-calcium metabolism disorders, most often resulting in neuropsychiatric symptoms. In our study, the discovery of this syndrome in our two patients was fortuitous thanks to the typical radiological appearance, associated with the clinical picture they initially presented, hence the interest of brain imaging in the diagnosis of this syndrome.
\end{abstract}

Keywords: Fahr syndrome; Cerebral calcification; Dysparathyroidism; Phosphocalcic

\section{Introduction}

Fahr syndrome is defined by the association of symmetrical non-arteriosclerotic calcifications of the basal ganglia, neuropsychiatric symptoms and parathyroid gland dysfunction, first and foremost is primary or postoperative hypoparathyroidism [1-4]. It is a rare anatomo-clinical entity whose pathophysiological mechanisms are controversial [5-7]. Defined by Theodor Fahr in 1930, Fahr's syndrome is distinguished from Fahr's disease (which is the primary genetic or sporadic form), by the presence of cerebral calcifications without disturbances in phosphocalcic metabolism [8]. We report two family pediatric cases hospitalized for afebrile convulsions, memory and neuropsychiatric disorders revealing Fahr syndrome secondary to pseudo-hypoparathyroidism.

\section{Case report and results}

F and W, brother and sister aged 13 and 16 years old respectively at the time of diagnosis, with no particular history, both admitted for management of repetitive, generalized tonic-clonic seizures lasting less than 5 min occurring in a context of apyrexia with retrograde amnesia, associated in the sister with a depressive syndrome. The physical examination in both children was normal apart from retrograde amnesia, with depressive syndrome in the sister only. Therefore, a para-clinical assessment was requested in the two patients who objectified:

- For the brother F. : hypocalcemia at $47 \mathrm{mg} / \mathrm{l}$, hyperphosphatemia at $99 \mathrm{mg} / \mathrm{l}$, normal magnesemia at $1.82 \mathrm{mg} / \mathrm{l}$, and parathyroid hormone (PTH) elevated at $678 \mathrm{pg} / \mathrm{ml}$. The electroencephalogram (EEG) has demonstrated a slowdown in background activity with the presence of diffuse intercritical anomalies predominantly in the temporo-occipital region. Brain computed tomography (CT) showed the presence of bilateral and symmetrical calcifications of the basal ganglia and the gray matter and white matter junction predominantly at the frontal level (Figure 1). In addition, the impact assessment of hypercalcemia, including standard x-rays of the hand (Figure 2), pelvis and dorsolumbar spine, renal ultrasound and ophthalmologic examination were normal.

\footnotetext{
* Corresponding author: Karima Larbi Ouassou

Department of pediatrics- Mohammed V Military Instruction Hospital - Rabat, Morocco.

Copyright $(2021$ Author(s) retain the copyright of this article. This article is published under the terms of the Creative Commons Attribution Liscense 4.0.
} 
World Journal of Advanced Research and Reviews, 2021, 12(01), 268-274

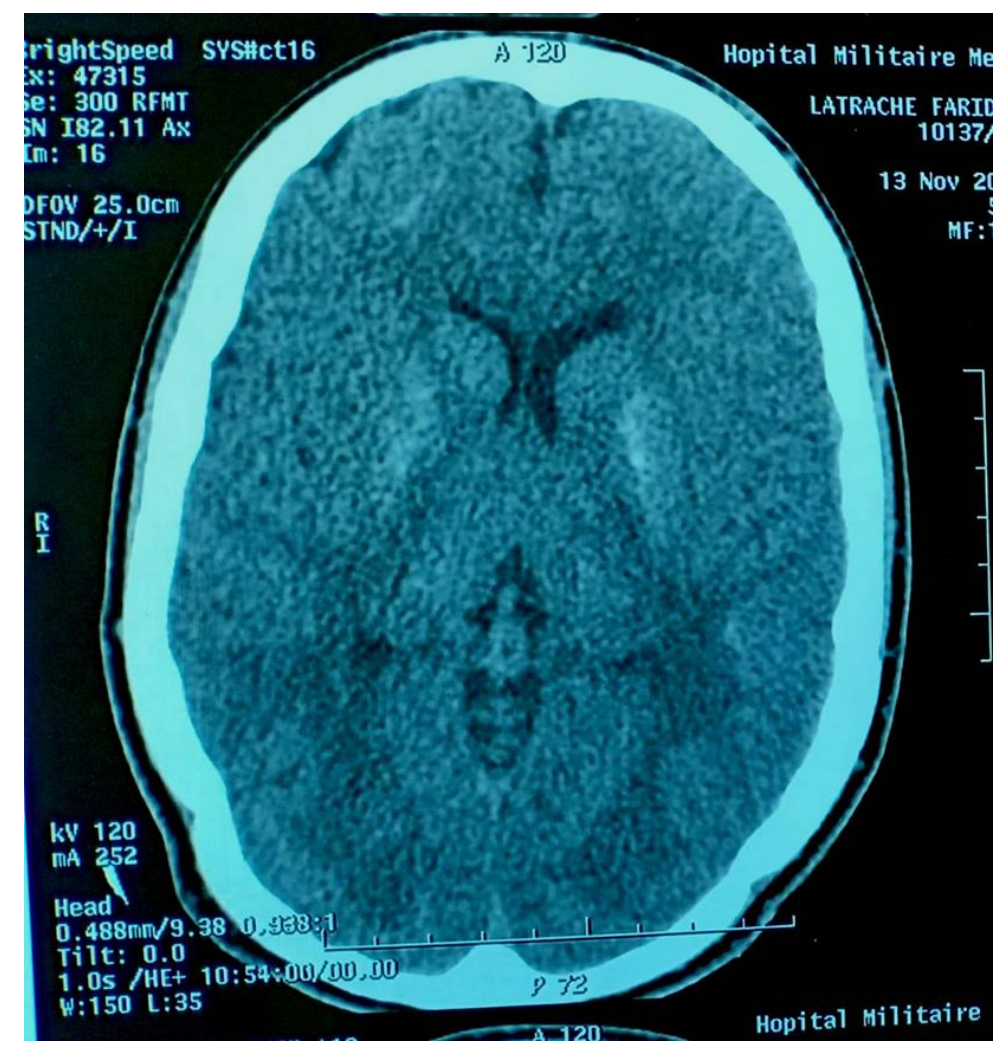

Figure 1: Cross-section of brother F's cerebral CDT showing calcifications of the basal ganglia

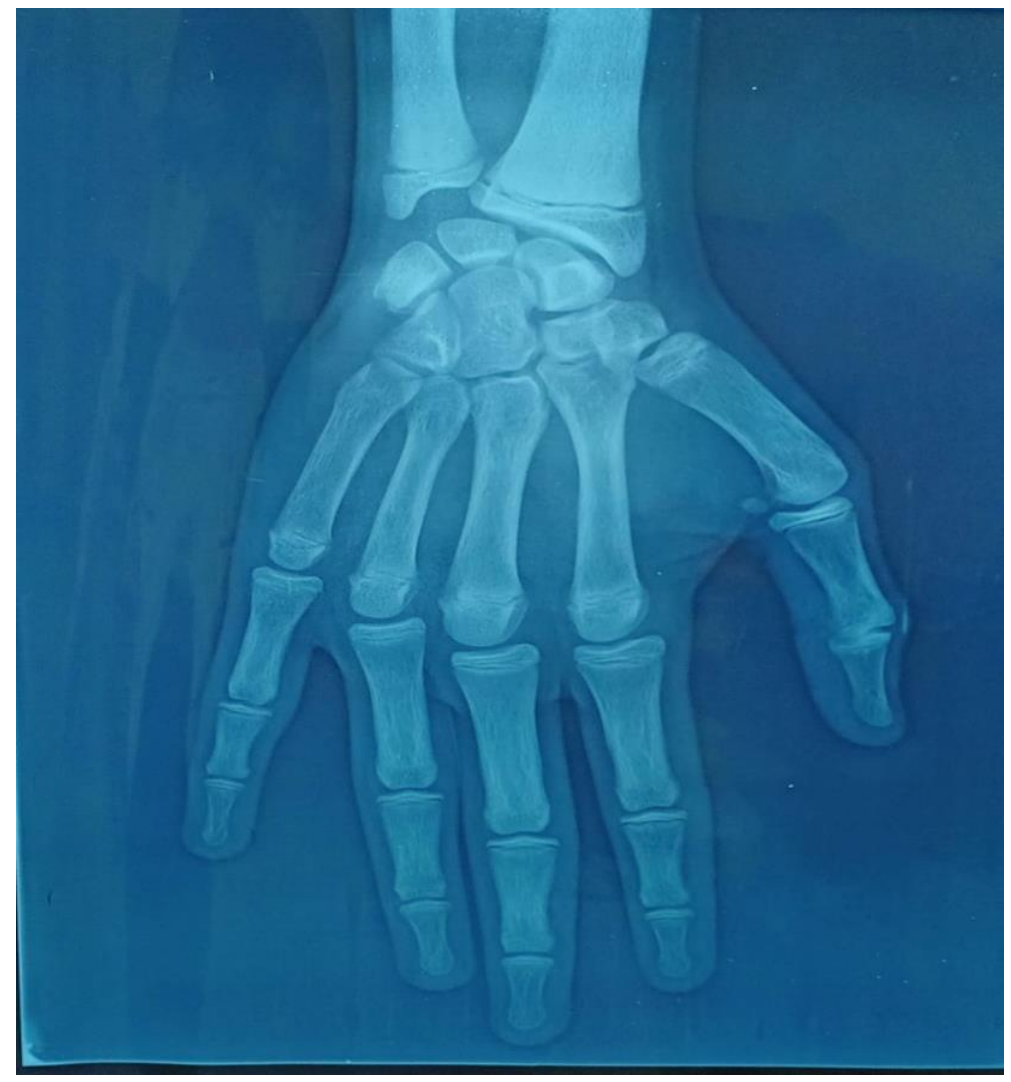

Figure 2 Standard x-ray of brother F's hand 
- For the sister W. : Hypocalcaemia at $70 \mathrm{mg} / \mathrm{l}$, normal phosphoremia at $43 \mathrm{mg} / \mathrm{l}$, PTH elevated at $327.4 \mathrm{pg} / \mathrm{ml}$ and hypokalaemia at $2.3 \mathrm{mEq} / \mathrm{l}$. The urinary ionogram and the exploration of the thyrotropic and corticotropic axes were all normal. The EEG and the brain CT (Figure 3) had showed the same aspect as her brother. In addition, the impact assessment of hypercalcemia, in particular standard x-rays of the hand (Figure 4), pelvis and dorsolumbar spine as well as the ophthalmologic examination were normal; the renal ultrasound noted the non-visualization of the right nothing, which appeared rudimentary on abdominal-pelvic CT.

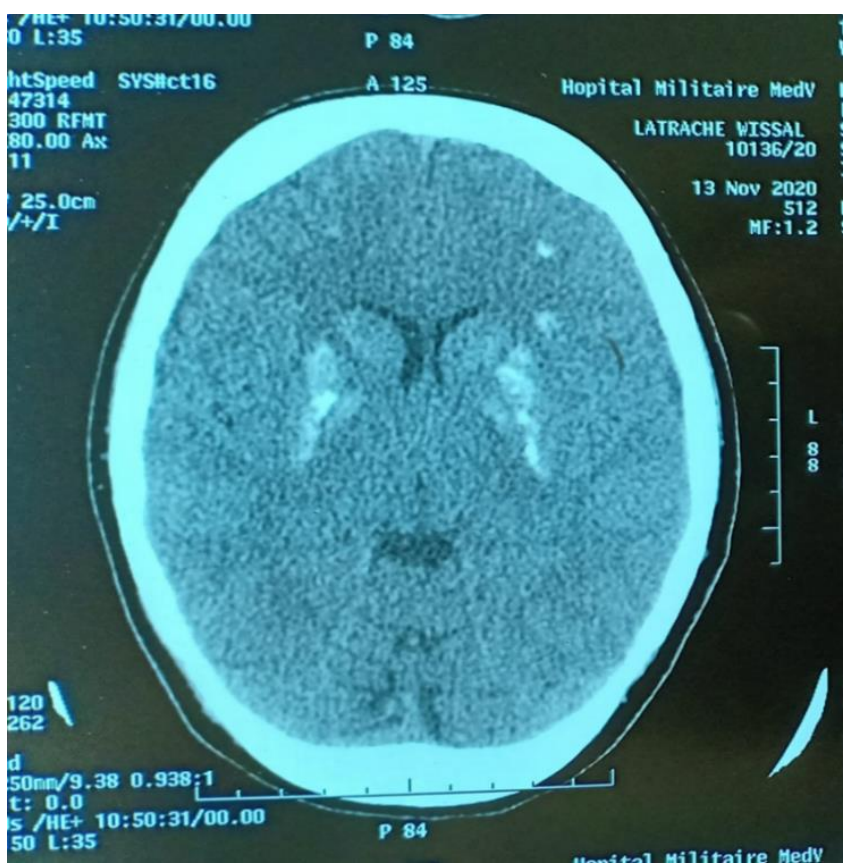

Figure 3 Cross-section of sister W's cerebral CDT showing calcifications of the basal ganglia

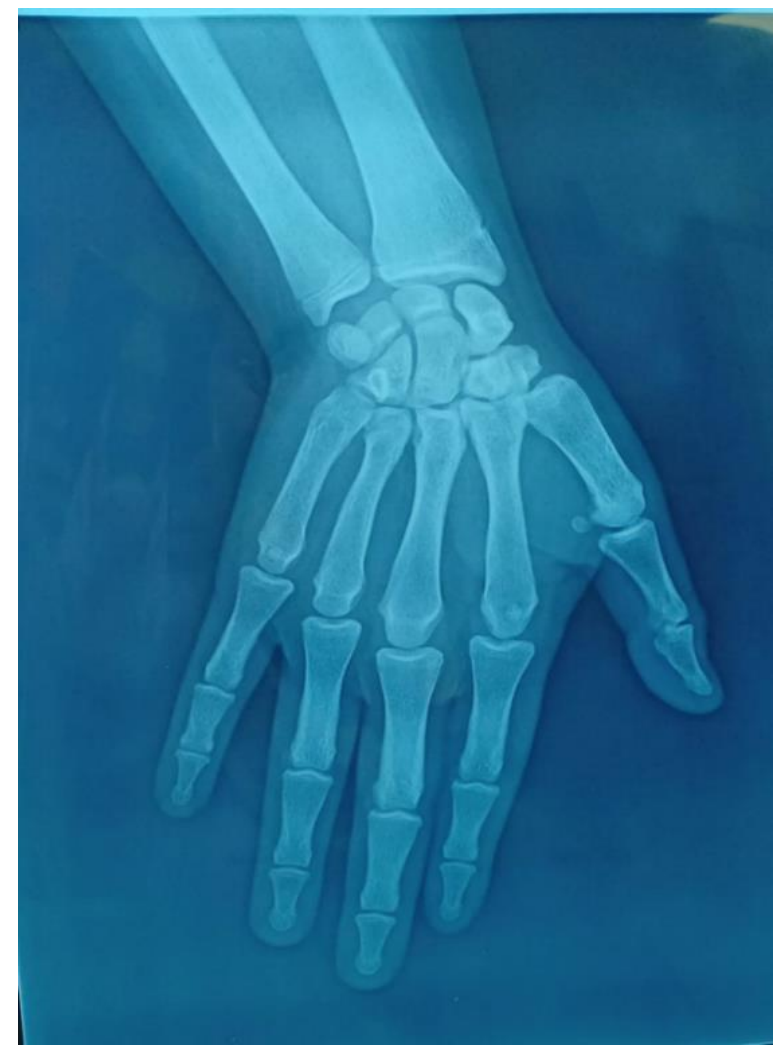

Figure 4 Standard $\mathrm{x}$-ray of brother F's hand 
At the end of clinical and para-clinical data, in particular scannographic data, the diagnosis of Fahr syndrome was retained, being secondary to pseudo-hypoparathyroidism. Therapeutically, the two patients were placed on bolus and orally, on cholecalciferol, on oral potassium and magnesium, and on full dose Sodium Valproate. The evolution was marked by the correction of the fluid and electrolyte disturbances, by the disappearance of convulsive seizures for the brother and by the reduction of the depressive syndrome for the sister.

\section{Discussion}

Fahr syndrome is a rare entity, the exact incidence of which is unknown, but it is estimated to be $<1 / 1000000$ [9]. It associates bilateral and symmetrical intracerebral calcifications of the central gray nuclei (NGC) mainly, of the serrated nuclei of the cerebellum and of the cerebral white matter with phosphocalcic metabolic disturbances $[8,10]$.

Several authors have preceded Theodor Fahr in the description of intracerebral calcifications, and this in various clinical situations: Delacour, in 1850, observed calcifications of the cerebral vessels in a patient with a tremor. . Bamberger (1855) described calcifications of small intracerebral vessels in a woman with mental retardation and epilepsy. Pick (1902) reported a case of tetany with calcifications of NGCs. Finally, Geyling and Penfield (1929) were the first to describe familial intracerebrals $[11,12]$.

The pathophysiology of Fahr syndrome is not fully understood. Dysfunction of a gene located on chromosome 14 results in calcium and fat deposits in demyelinated areas [13]. Most authors suggest a metabolic disorder of oligoglial cells with deposits of mucopolysaccharides and secondary onset vascular and perivascular lesions and calcareous encrustations $(8,11)$. Indeed, the post-mortem histochemical analysis of patients with this syndrome has objectified on a microscopic scale of basophilic mineral deposits in the vascular walls (arteries, capillaries and venous): calcium, iron, zinc, copper, magnesium, aluminum. And potassium. While the intima of these vessels is preserved. Thus, the term NGC mineralization would be more appropriate to describe this entity, calcium not being the only element involved in the genesis of deposits $[2,14]$.

Clinically, Fahr syndrome is generally difficult to suspect because it can remain asymptomatic or cause manifestations which do not correspond to any specific picture, without obvious anatomo-clinical correlation $[5,8,15]$. In the literature, clinical manifestations primarily include neuropsychiatric signs such as character disorders, mood disorders (manic access, depression as in our patient) and / or behavior, or even a confusional or delusional syndrome, distortions perceptual, auditory and visual hallucinations $[11,12,16,17]$. Other neurological manifestations are possible but less usual, such as cognitive disorders, in particular memory disorders and lack of concentration as well as mental debility $[3,5,8]$ observed elsewhere in our two patients. The manifestations can sometimes be summed up in signs of hypocalcemia caused by dysparathyroidism (tetany, Chvostek's sign, trousseau sign) [18,19] an extrapyramidal syndrome, or more rarely, cerebellar or pyramidal syndrome, intracranial hypertension syndrome, damage to the cranial nerves or abnormal movements which are classically frequent in pediatric forms but not noticed in our two patients [19]. A picture of progressive supranuclear palsy has also been reported. Partial or generalized seizures are less common but may be the main warning sign [10]. Moreover, they presented the reason for hospitalization with the brother, while the sister presented a depressive syndrome long unrecognized by the parents. Nevertheless, FS can remain asymptomatic [11, 15, 20,21] and can only develop in adulthood as described by E. Turki et al [22].

Before the advent of the digital scanner, the para-clinical diagnosis of SF was based on cranial x-rays and autopsy (19). Currently The diagnostic examination of choice is cerebral computed tomography for the detection of intracerebral calcifications [23-27] These are typically bilateral and symmetrical concerning the central gray nuclei, more frequently the striated nuclei, the thalamus, the serrated nuclei, the semi-oval center $(18,28)$. However, it is important not to confuse SF with other conditions that can cause intracerebral calcifications, in particular endocrinopathies (hypothyroidism, hypogonadism), systemic pathologies (systemic scleroderma, systemic lupus erythematosus, celiac disease) infections (toxoplasmosis, neurocysticercosis, rubella), various diseases (chronic renal failure, vitamin D intoxication, mitochondrial cytopathies) and primary or secondary calcified brain tumors. However, the intracerebral calcifications observed during these different pathologies have different sites and aspects (19) the value of magnetic resonance imaging (MRI) remains limited in the context of Fahr syndrome.. It usually shows calcifications which appear as hyper signals in T1 and T2, sometimes as hypointense in T1 (18). As for functional imaging, in particular the SPECT scanner (single proton emission computed tomography), it allows a better understanding of the physiopathological mechanisms at the origin of SF by objectifying perfusion anomalies in calcified regions whereas PET scanner (Positron emission tomography scan) did not find any significant difference [29] but access to these exams is still not possible in our context. 
In the absence of clear pathophysiological evidence, it remains difficult to define the cause and effect link to define the exact origin of Fahr syndrome. Nevertheless, according to several studies, hypoparathyroidism remains the most classic cause of Fahr syndrome, whether primary or postoperative. (15). It associates hypocalcemia, hyperphosphatemia, hypocalciuria and a decrease in the serum parathyroid hormone level explaining the majority of clinical signs of SF $[17,30]$.

Pseudo-hypoparathyroidism, often familial, is also a common cause associated with SF, although it is rare in children and remains asymptomatic for a long time $(15,20,31)$ N. Kahloul et al. reported a case in 2009 in a 12-year-old Tunisian child, and Y. Sekkach et al. reported a second case in 2011 in a 12-year-old Moroccan child . This phosphocalcic disorder produces a picture similar to hypoparaphyroidism with normal or elevated parathyroid hormone; (32, 33); this is also the case with our two patients. Hyperparathyroidism has been exceptionally reported as a cause of this syndrome [15, $20,31]$.

In contrast to the severity of the symptoms for which it may be responsible, SF has a good prognosis and the correction of disorders of phosphocalcic metabolism often brings about a notable improvement. Indeed, calcivitaminotherapy $\mathrm{D}$ is the basis of the treatment of SF $(2,12,17)$. This is the case of our two patients, moreover, who presented a spectacular improvement under calcium supplementation and in vitamin D. According to the data in the literature, treatment with specific blockers of intracerebral calcium channels has been tried without success. Disodium etidronate would also have a beneficial effect on clinical signs in a double-blind study, compared to placebo (34). However, if, despite the correction of these biological disturbances, neurological or neuropsychiatric manifestations persist, patients should benefit from symptomatic treatments. In the event of initiality, antiepileptic drugs may be offered while avoiding those which may induce or worsen hypocalcemia [35-37]. Anxiolytic drugs, antidepressants and antipsychotics may be prescribed in the event of persistent neuropsychiatric disorders $(35,36)$. In addition, progressive cognitive impairment requires multidisciplinary management (neurologist, pediatric neurologist, neuro-psychologist, and psychomotor therapist, speech therapist) in order to improve the living conditions of these patients and their families.

\section{Conclusion}

Fahr syndrome is a rare anatomo-clinical and radiological entity, with multiple clinical and etiological aspects, the pathophysiological mechanism of which remains poorly understood, and the treatment is simple and effective, sometimes contrasting with the severity of the symptoms. Our work reports the benefit of systematically carrying out a phosphocalcic assessment in front of cerebral calcifications or neuro-psychiatric manifestations in children. The genetic study, for its part, is required in front of SF with negative results, as similar familial cases may be part of Fahr's disease or in front of a hereditary metabolic disorder.

\section{Compliance with ethical standards}

\section{Acknowledgments}

Thanks to all my supervisors for providing guidance and feedback throughout this project.

\section{Disclosure of conflict of interest}

None

\section{Statement of informed consent}

Informed consent was obtained from all individual participants included in the study.

\section{References}

[1] Fahr T. Idiopathic calcification of the cerebral vessels. Zentralbl general pathol. 1930-1931; 50: 129-33.

[2] Morgante L, Trimarchi F, Benvenga S. Fahr's disease. Lancet. 2 Mar 2002; 359(9308): 759.

[3] Chouaib N, Rafai M, Belkouch A, Bakkali H, Belyamani L. Fortuitous discovery of Fahr syndrome following a seizure.Rev Neurol. 2015; 171: 894-5.

[4] El Hechmi S, Bouhlel S, Melki W, El Hechmi Z. Psychotic disorder secondary to Fahr syndrome: about an observation. Encephale. 2014; 40: 271-5. 
[5] Chevalier D, Marie I, Tillon J, Lévesque H. A cause of intracerebral calcifications that should not be ignored: Fahr syndrome. Rev Med Interne. 2005; 26: 668-77.

[6] Somaï M, Chaker F, Belhadj L, Yazidi M, Chihaoui M, Rejeb O, et al. Fahr syndrome secondary to hypoparathyroidism: clinical and radiological presentation. Rev med interne. 2016; 37: A170.

[7] Cassiani-Miranda CA, Herazo-Bustos M, Cabrera-Gonzalez A, Cadena-Ramos I, Barrios-Ayola F. Psychosis associated with Fahr's syndrome: Acase report. Rev Colomb Psiquiatr. 2015; 44: 256-61.

[8] Khammassi N, Chrifi J, Mohsen D, Abdelhedi H, Tougourti MN, HamzaM. Fahr syndrome: about two cases. Rev Neurol. 2010; 166: 446-50.

[9] Sridana ME, Sukmana Putra S. Fahr's Syndrome: a rare case report. Indo-nesia J Biomed Sci. 2016 ; 10 : 15-7.

[10] Hmami F, Chaouki S, Benmiloud S, Souilmi FZ, Abourazzak S, Idrissi M,et al. Epileptic attacks revealing abnormalities of the phospho-calcium metabolism. Rev Neurol. 2014; 170: 440-4.

[11] El Maghraoui A, Birouk N, Zaim A. Fahr syndrome and dysparathyroidism. 3 cases. Presse Med. 30 Sep 1995; 24(28): 1301-4.

[12] El Hechmi, Bouhlel S, Melki W, El Hechmi Z. Psychotic disorder secondary to Fahr syndrome: about an observation. Encephale. Jun 2014; 40(3): 271-5.

[13] Beall SS, Patten BM, Mallette L. Abnormal systemic metabolism of iron, porphyrin and calcium in Fahr's syndrome. Ann Neurol 1989; 26: 569-75.

[14] Finsterer J. Mitochondriopathies. Eur J Neurol. Mar 2004; 11(3): 163-86.

[15] Sbai H, Smail L, Hamdani S, Essatara Y, Harrandou M, Khatouf M, et al. Fahr syndrome discovered after pneumococcal meningitis. Rev Med Interne. 2008; 29: 412-4.

[16] Jochim K, Kucharski R, Wozniak M, Nowak M, Łachut K, Ziółkowska-Kochan M, et al. Cognitive impairment and psychopathologicalsymptoms in Fahr's syndrome. Neuropsychiatria i Neuropsycholo-gia/Neuropsychiatry and Neuropsychology. 2010; 5: 90-4.

[17] Otheman Y, Khalloufi H, Benhima I, Ouanass A. Neuro-psychiatric manifestations revealing pseudohypoparathyroidism with Fahr syndrome. Encephale. 2011; 37: 54-8.

[18] Casanova MF. Araque JM. Mineralization of the basal ganglia: implica-tions for neuropsychiatry, pathology and neuroimaging. Psychiatry Res. 2003; 12: 59-87.

[19] Rafaia, ,b, S. Oumaria, S. Lytima, F.Z. Boulaajajc, B. El Moutawakkila, I. Slassia Fahr's syndrome: clinical, radiological and etiological aspects; 0181-9801X/\$ - 2013 Publie' par Elsevier Masson SAS. Feuillets de radiologie. 2014; 54: 2-8.

[20] Noyelle C, Bruet A, Molhob M, Veyssier-Belot C. Confusion syndrome. Rev Med Interne. 2008; 29: 500-1.

[21] Duron E, Seux ML, De Rotrou, Rigaud AS. Fahr syndrome expressed by a picture of progressive supranuclear palsy. Rev Neurol. 2007; 163: 161-2.

[22] Khadir K, Moussaid L, el Ouazzani T, et al. Fahr syndrome secondary to hypoparathyroidism with dermatological revelation. Ann Dermatol Venereol. 2004; 131: 979-83.

[23] Faria AV, Pereira IC, Nanni L. Computerized tomography findings in Fahr's syndrome. Arq Neuropsiquiatr. 2004; 62(3- B): 789-92.

[24] Brannan TS, Burger AA, Chaudhary MY. Bilateral basal ganglia calcifications visualise don CT scan. J Neurol Neurosurg Psychiatry. 1980; 43: 403-6.

[25] Fenelon G, Gray F, Paillard F, Thibierge M, Mahieux F, Guillard A. A prospective study of patients with CT detected pallidal calcifications. J Neurol Neurosurg Psychiatry. 1993; 56: 622-5.

[26] Midassi O, Hammami H, Somaï M, Fenniche S. Fahr syndrome revealed by pustular psoriasis: a case report. Rev Med Interne. 2016; 37: A170.

[27] Gülsün M, Fuat Bayk'yz A, Kabata S, Belli H. Fahr's Syndrome - Threecases presenting with psychiatric signs. Eur J Gen Med. 2006; 3: 35-40.

[28] El Boukhrissi F, Zoulati G, En-nafaa I, Ouleghzal H, Derrou S, Safi S, et al. Fahr syndrome secondary to primary hypoparathyroidism: a case report. Pan African Med J. 2017; 26: 2. 
[29] Manyam BV. What is and what is not "Fahr's disease". Parkinsonism Relat Disord. 2005; 11: 73-80.

[30] Rharrabti S, Darouich I, Benbrahim M, Belahsen F, Rammouz I, AlouaneR. Confusional syndrome revealing Fahr syndrome with hyper-parathyroidism. Pan African Med J. 2013; 14: 123.

[31] Faissolle P, Wagner-Mahler K, Mas JC, Moreigne M, Khalfi A, Leonetti V, et al. A cause of intracerebral calcifications not to be overlooked: pseudo-hypoparathyroidism type IB. Arch Pediatr. 2008; 15: 1433-6.

[32] A. ES SEDDIKI*, A. AYYAD*, N. BENAJIBA*, H. HADJ KACEM**, Fahr syndrome and pseudohypoparathyroidism pediatrie-pratique.com/journal/article/0011667-syndrome-de-fahr-et-pseudohypoparathyroidie. 28 June 2016.

[33] Kahloul N, Chaari W, Boughamoura L, Charfeddine L, Khammeri S, AmriF. A Fahr syndrome indicative of pseudohypoparathyroidism. ArchPediatr. 2009; 16: 444-8.

[34] Loeb JA, Sohrab SA, Huq M, Fuerst DR. Brain calcifications induce neurological dysfunction that can be reversed by a bone drug. J Neurol Sci. 2006; 243: 77-81.

[35] Jaworski K, Styczy'nska M, Mandecka M, Walecki J, Kosior DA. Fahrsyndrome-an important piece of a puzzle in the differential diagnosis ofmany diseases. Polish J Radiol. 2017; 82: 490-3.

[36] Marinkovi'c DM, Dragovi'c T, Kikovi'c S, Kuzmi'c Jankovi'c S, Djuran Z,Zoran Hajdukov Z. Fahr's syndrome and idiopathic hypoparathyroidism-Acase report. Vojnosanit Pregl. 2017; 74: 184-8.

[37] Chaabène A, Kamoun F, Charfi N, Triki C, Abid M, Mhiri C. Hypocalcemic seizures: a study of 7 cases. Epilepsies. 2006; 18: 149-54 